\title{
A RESIDUAL-FREE BUBBLE FORMULATION FOR NONLINEAR ELLIPTIC PROBLEMS WITH OSCILLATORY COEFFICIENTS
}

\author{
MANUEL BARREDA \\ Departamento de Matemática, Universidade Federal do Paraná, Curitiba - PR, Brazil
}

\author{
ALEXANDRE L. MADUREIRA \\ Laboratório Nacional de Computação Científica, Petrópolis - RJ, Brazil \\ Fundação Getúlio Vargas, Rio de Janeiro - RJ, Brazil
}

\begin{abstract}
We present an investigation of the Residual Free Bubble finite element method for a class of multiscale nonlinear elliptic partial differential equations. After proposing a nonlinear version for the method, we address fundamental questions as existence and uniqueness of solutions. We also obtain a best approximation result, and investigate possible linearizations that generate different versions for the method. As far as we are aware, this is the first time that an analysis for the nonlinear Residual Free Bubble method is considered.
\end{abstract}

\section{INTRODUCTION}

Important physics and engineering problems are nonlinear and of multiscale nature. Examples include certain models for flow in porous media and mechanics of heterogeneous materials. We consider in this work nonlinear elliptic problems of the form

$$
-\operatorname{div}\left[a_{\epsilon}\left(x, u_{\epsilon}, \nabla u_{\epsilon}\right)\right]=f \quad \text { in } \Omega, \quad u_{\epsilon}=0 \quad \text { on } \partial \Omega,
$$

where $\Omega \subset \mathbb{R}^{2}$ is a polygonal domain,

$$
a_{\epsilon}\left(x, u_{\epsilon}, \nabla u_{\epsilon}\right)=\alpha_{\epsilon}(x) b\left(u_{\epsilon}\right) \nabla u_{\epsilon} .
$$

and $\alpha_{\epsilon}$ might have an oscillatory nature. We describe further restrictions on the coefficients latter on.

E-mail addresses: barreda@mat.ufpr.br, alm@lncc.br, alexandre.madureira@fgv.br.

Date: March 24, 2017.

Key words and phrases. Multiscale PDE; Finite Element Method; Nonlinear elliptic PDE; Numerical analysis; Residual Free Bubbles.

Research of the second author was supported by CNPq, Brazil. 
Problems like (1.1) are often dealt with using homogenization techniques, even in the linear case. However, this is not always convenient due to restrictive hypothesis on the coefficients, like periodicity or certain probabilistic distributions. Thus, even for the linear situation, several authors developed methods that can compute approximations that do not rely on homogenization.

It is well-known that standard Galerkin methods perform poorly for such equations, linear or nonlinear, under the presence of oscillatory coefficients [13,23], and there is a strong interest in developing numerical schemes that are efficient for problems with multiscale nature. Important methods include the Generalized Finite Element Method (GFEM) [7], the Discontinuous Enrichment Method (DEM) [28], the Heterogeneous Multiscale Method (HMM) [21], and the Multiscale Hybrid Mixed Method (MHM) [2, 32, 36]. We concentrate our literature review on the the Residual-Free Bubble Method (RFB) [8, 13, 15, 29, 30] and the Multiscale Finite Element Method (MsFEM) [23, 26, 34, 35, 37] since they are closer to our own method. For all the above methodology, the goal is to derive numerical approximations for the multiscale solution using a mesh that is coarser than the characteristic length $\epsilon$ of the oscillations (in opposition to [43, 44]).

The idea behind the MsFEM is to incorporate local information of the underlying problem into the basis functions of the finite element spaces, capturing microscale aspects. Its analysis was first considered for linear problems, and assuming that the coefficients of the equations are periodic [25,35]. Latter, the non periodic case was also considered [33]. An extension for nonlinear problems appears in [24], for pseudo-monotone operators, and the authors show that, under periodicity hypothesis, the numerical solution converges towards the homogenized solution. They also determine the convergence rate if the flux depends only on the gradient of the solution. Further variations of the method were considered in [17, 18]. The MHM method shares some of the characteristics of the MsFEM, but so far it was considered only for linear problems.

The HMM approach for linear and nonlinear problems differs considerably, but, as in the MsFEM, the method is efficient in terms of capturing the macroscale behavior of multiscale problems. See [21,38] for a description of the method, and 22] for a analysis of the method involving linear and nonlinear cases.

The Residual Free Bubble (RFB) formulation [8, 14, 15] was first considered with advectionreaction-diffusion problems in mind. The use of RFB for problems with oscillatory coefficients was already suggested in [13, and investigated in 41] for the linear case. See [46] for a clear description of how the MsFEM and RFB relate.

In the present work, we extend the RFB formulation for a class of nonlinear problems, with oscillatory coefficients, as in (1.1). Such model is a natural extension of the linear problem with oscillatory coefficients, and of the nonlinear problems as considered in [20], without oscillatory coefficients. We remark that the RFB was considered only in the linear setting, with one exceptions [40] which considers numerical experiments with RFB for shallow water problem in an ad hoc manner. 
Assume that $\alpha_{\epsilon}():. \Omega \rightarrow \mathbb{R}$ is measurable, and that there exist positive constants $\alpha_{0}$ and $\alpha_{1}$ such that

$$
0<\alpha_{0} \leq \alpha_{\epsilon}(x) \leq \alpha_{1} \text { almost everywhere in } \Omega .
$$

Assume also that $b: \mathbb{R} \rightarrow \mathbb{R}$ is continuous and belongs to $W^{2, \infty}(\mathbb{R})$, and that there exists a constant $b_{0}$ such that

$$
0<b_{0} \leq b(t) \quad \text { for all } t \in \mathbb{R} .
$$

Note that a uniform coercivity follows from the above hypothesis, i.e., for almost every $x \in \Omega$, and all $t \in \mathbb{R}$ and $\boldsymbol{\xi} \in \mathbb{R}^{2}$,

$$
\alpha_{\epsilon}(x) b(t) \boldsymbol{\xi} . \boldsymbol{\xi} \geq \alpha_{0} b_{0}\|\boldsymbol{\xi}\|^{2} .
$$

Rewriting (1.1) in its variational formulation, we have that $u_{\epsilon} \in H_{0}^{1}(\Omega)$ solves

$$
a\left(u_{\epsilon}, v\right)=(f, v) \text { for all } v \in H_{0}^{1}(\Omega),
$$

where

$$
a(\psi, \phi)=\int_{\Omega} \alpha_{\epsilon}(x) b(\psi) \nabla \psi \cdot \nabla \phi d x .
$$

Throughout this paper, we denote by $L^{2}(\Omega)$ the space of square integrable functions, by $W^{q, p}, H_{0}^{1}(\Omega), H^{1}(\Omega)$ the usual Sobolev Spaces, and by $H^{-1}(\Omega)$ the dual space of $H_{0}^{1}(\Omega)[12$, 27. By $C$ we denote a generic constant that might have different values at different locations, but that does not depend on $h$ or $\epsilon$.

The outline of the article is as follows. After the introductory Section 1, we describe the RFB method in Section 2, and discuss existence and uniqueness of solutions in Section 3, A best approximation result is obtained in Section 4, and possible linearizations are discussed in Section 5 ,

\section{The Residual Free Bubble Method}

Let $\mathcal{T}_{h}=\{K\}$ be a partition of $\Omega$ into finite elements $K$, and, associated to $\mathcal{T}_{h}$, the subspace $V_{h} \subset H_{0}^{1}(\Omega)$ of piecewise polynomials. The classical finite element Galerkin method seeks a solution of (1.4) within $V_{h}$. The RFB method seeks a solution within the enlarged, or enriched, space $V_{r}=V_{h} \oplus V_{b}$, where the bubble space is given

$$
V_{b}=\left\{v \in H_{0}^{1}(\Omega):\left.v\right|_{K} \in H_{0}^{1}(K) \text { for all } K \in \mathcal{T}_{h}\right\} .
$$

That means that we seek $u_{r} \in V_{r}$ such that

$$
\int_{\Omega} \alpha_{\epsilon}(x) b\left(u_{r}\right) \nabla\left(u_{r}\right) \cdot \nabla v_{r} d x=\int_{\Omega} f v_{r} d x \quad \text { for all } v_{r} \in V_{r} .
$$

The second equation in the above system is obtained, for each fixed element $K$, by considering $\left.v_{r}\right|_{K} \in H_{0}^{1}(K)$ arbitrary and vanishing outside $K$. An integration by parts yield the strong equation of (2.1). 
This is equivalent to search for $u_{r}=u_{h}+u_{b}$, where $u_{h} \in V_{h}$ and $u_{b} \in V_{b}$ solve

$$
\begin{gathered}
\int_{\Omega} \alpha_{\epsilon}(x) b\left(u_{h}+u_{b}\right) \nabla\left(u_{h}+u_{b}\right) \cdot \nabla v_{h} d x=\int_{\Omega} f v_{h} d x \quad \text { for all } v_{h} \in V_{h}, \\
-\operatorname{div}\left[\alpha_{\epsilon}(x) b\left(u_{h}+u_{b}\right) \nabla\left(u_{h}+u_{b}\right)\right]=f \quad \text { in } K, \text { for all } K \in \mathcal{T}_{h} .
\end{gathered}
$$

The coupled system (2.2) defines the Residual Free Bubble Method. The use of bubbles allows the localization of the problems of the second equation of (2.2), while the first equation has a global character. Such formulation induces a two-level discretization, where the global problem given by the first equation in (2.2) should be discretized by a coarse mesh, and the local problems given by the second equation of (2.2) should be solved in a fine mesh. Thus, in terms of computational cost, the first equation is global but posed in a coarse mesh, and the second equation requires refined meshes, but they are local and can be solved in parallel.

Note that for linear problems, it is possible to perform static condensation, "eliminating" the bubble part in the final formulation, which is then modified and posed only on the polynomial space [8, 13, 15, 31, 41]. See remark below.

Remark 2.1. If $\mathcal{L}$ denotes a linear differential operator, and $a(\cdot, \cdot)$ the associated bilinear form, then it results from the $R F B$ that $u_{b} \in H_{0}^{1}(K)$ solves

$$
\mathcal{L} u_{b}=-\mathcal{L} u_{h}+f \quad \text { in } K \text {. }
$$

Denoting by $\mathcal{L}_{K}^{-1}: H^{-1}(K) \rightarrow H_{0}^{1}(K)$ the local solution operator, we gather that $\left.u_{b}\right|_{K}=$ $\mathcal{L}_{K}^{-1}\left(f-\mathcal{L} u_{h}\right)$. Thus $u_{h} \in V_{h}$ solves that

$$
a\left(u_{h}, v_{h}\right)+a\left(\sum_{K \in \mathcal{T}_{h}} \mathcal{L}_{K}^{-1} \mathcal{L} u_{h}, v_{h}\right)=\left(f, v_{h}\right)-a\left(\sum_{K \in \mathcal{T}_{h}} \mathcal{L}_{K}^{-1} f, v_{h}\right) \quad \text { for all } v_{h} \in V_{h} .
$$

The formulation above is a perturbed Galerkin formulation. The perturbation aims to capture the microscale effects neglected by coarse meshes.

\section{Existence And Uniqueness of Solutions}

In this section we prove existence and uniqueness results for the continuous problem and for the RFB formulation. We adapt here ideas present in [4,11]. We shall make use of the following version of the Schauder Fixed-Point Theorem [19].

Theorem 3.1 (Schauder Fixed-Point Theorem). Let $E$ be a normed space, $A \subset E$ a nonempty convex set, and $C \subset A$ compact. Then, every continuous mapping $T: A \rightarrow C$ has at least one fixed point.

The following result guarantees existence and uniqueness of solutions for the variational problem (1.4).

Theorem 3.2. Let $\alpha_{\epsilon}($.$) and b($.$) such that (1.2) and (1.3) hold. Then, given f \in L^{2}(\Omega)$, the variational problem (1.4) has one and only one solution in $H_{0}^{1}(\Omega)$. 
Our proof of Theorem 3.2 is based on the lemmata that follow. We first observe that (1.5) suggests the definition

$$
T^{\epsilon}: L^{2}(\Omega) \rightarrow H_{0}^{1}(\Omega),
$$

such that, for every $w \in L^{2}(\Omega)$, the operator $w^{\epsilon}=T^{\epsilon}(w) \in H_{0}^{1}(\Omega)$ solves

$$
\int_{\Omega} \alpha_{\epsilon}(x) b(w) \nabla w^{\epsilon} . \nabla v d x=\int_{\Omega} f v d x \quad \text { for all } v \in H_{0}^{1}(\Omega) .
$$

The operator $T^{\epsilon}$ is clearly well-defined since, from the hypothesis imposed on $\alpha_{\epsilon}$ and $b$, the bilinear form above satisfies the hypothesis of Lax-Milgram Lemma.

Lemma 3.3. Under the hypothesis of Theorem [3.2, the operator $T^{\epsilon}$ given by (3.1) is continuous.

Proof. Let $\left\{w_{m}\right\}$ be a sequence in $L^{2}(\Omega)$ such that $w_{m} \rightarrow w$ strongly in $L^{2}(\Omega)$. Consider $T^{\epsilon}\left(w_{m}\right)=w_{m}^{\epsilon}$ and $T^{\epsilon}(w)=w^{\epsilon}$. Then,

$$
\begin{gathered}
\int_{\Omega} \alpha_{\epsilon}(x) b\left(w_{m}\right) \nabla w_{m}^{\epsilon} \cdot \nabla v d x=\int_{\Omega} f v d x \quad \text { for all } v \in H_{0}^{1}(\Omega), \\
\int_{\Omega} \alpha_{\epsilon}(x) b(w) \nabla w^{\epsilon} \cdot \nabla v d x=\int_{\Omega} f v d x \quad \text { for all } v \in H_{0}^{1}(\Omega) .
\end{gathered}
$$

Subtracting both equations, it follows that

$$
\int_{\Omega} \alpha_{\epsilon}(x) b\left(w_{m}\right) \nabla w_{m}^{\epsilon} \cdot \nabla v d x-\int_{\Omega} \alpha_{\epsilon}(x) b(w) \nabla w^{\epsilon} \cdot \nabla v d x=0 \quad \text { for all } v \in H_{0}^{1}(\Omega) .
$$

Adding and subtracting $w^{\epsilon}$ we gather that

$$
\int_{\Omega} \alpha_{\epsilon}(x) b\left(w_{m}\right) \nabla\left(w_{m}^{\epsilon}-w^{\epsilon}+w^{\epsilon}\right) \cdot \nabla v d x=\int_{\Omega} \alpha_{\epsilon}(x) b(w) \nabla w^{\epsilon} \cdot \nabla v d x \quad \text { for all } v \in H_{0}^{1}(\Omega) .
$$

In an equivalent form, for each $v \in H_{0}^{1}(\Omega)$,

$$
\int_{\Omega} \alpha_{\epsilon}(x) b\left(w_{m}\right) \nabla\left(w_{m}^{\epsilon}-w^{\epsilon}\right) \cdot \nabla v d x=\int_{\Omega} \alpha_{\epsilon}(x)\left(b(w)-b\left(w_{m}\right)\right) \nabla w^{\epsilon} \cdot \nabla v d x .
$$

In particular, for $v=w_{m}^{\epsilon}-w^{\epsilon}$ it follows that

$$
\begin{gathered}
\alpha_{0} b_{0}\left\|\nabla\left(w_{m}^{\epsilon}-w^{\epsilon}\right)\right\|_{0, \Omega}^{2} \leq \int_{\Omega} \alpha_{\epsilon}(x) b\left(w_{m}\right) \nabla\left(w_{m}^{\epsilon}-w^{\epsilon}\right) \cdot \nabla\left(w_{m}^{\epsilon}-w^{\epsilon}\right) \\
=\int_{\Omega} \alpha_{\epsilon}(x)\left(b(w)-b\left(w_{m}\right)\right) \nabla w^{\epsilon} \cdot \nabla\left(w_{m}^{\epsilon}-w^{\epsilon}\right) d x \\
\leq \alpha_{1}\left\|\left[b(w)-b\left(w_{m}\right)\right] \nabla w^{\epsilon}\right\|_{0, \Omega}\left\|\nabla\left(w_{m}^{\epsilon}-w^{\epsilon}\right)\right\|_{0, \Omega}
\end{gathered}
$$

Thus, $\left\|\nabla\left(w_{m}^{\epsilon}-w^{\epsilon}\right)\right\|_{0, \Omega} \leq C\left\|\left[b(w)-b\left(w_{m}\right)\right] \nabla w^{\epsilon}\right\|_{0, \Omega}$. Now [4], since $b(w)-b\left(w_{m}\right) \rightarrow 0$ in measure, and that $\left|\nabla w^{\epsilon}\right|^{2} \in L^{1}(\Omega)$, we conclude that $\left\|\left[b(w)-b\left(w_{m}\right)\right] \nabla w^{\epsilon}\right\|_{0, \Omega} \rightarrow 0$. Thus $w_{m}^{\epsilon} \rightarrow w^{\epsilon}$ strongly in $H^{1}(\Omega)$. 
Lemma 3.4. Let $F \in C^{1}(\mathbb{R})$ such that $F(0)=0$ and $\left|F^{\prime}(t)\right| \leq L$ for all $t \in \mathbb{R}$. Let $\Omega \subset \mathbb{R}^{d}$ be open, and let $1 \leq p<\infty$. Then

a) if $v \in W^{1, p}(\Omega)$, then $F \circ v \in W^{1, p}(\Omega)$ and $\partial(F \circ v) / \partial x_{i}=F^{\prime}(v) \partial v / \partial x_{i}$, for $1 \leq i \leq d$

b) if $v \in W_{0}^{1, p}(\Omega)$, then $F \circ v \in W_{0}^{1, p}(\Omega)$.

Proof. [12, Proposition 9.5].

Lemma 3.5. Under the hypotheses of Theorem [3.2, the uniqueness of solutions for (1.1) follows.

Proof. Let, for $t \in \mathbb{R}$,

$$
\tilde{b}(t)=\int_{0}^{t} b(s) d s
$$

Since $b \in C^{0}(\mathbb{R})$, then $\tilde{b} \in C^{1}(\mathbb{R})$. Moreover, $\tilde{b}^{\prime}$ is always positive, and then $\tilde{b}$ is a bijection in $\mathbb{R}$. Consider the Kirchhoff transform $U_{\epsilon}=\tilde{b}\left(u_{\epsilon}\right)$. From Lemma 3.4 we gather that

$$
\nabla U_{\epsilon}=b\left(u_{\epsilon}\right) \nabla u_{\epsilon}
$$

and $U_{\epsilon} \in H_{0}^{1}(\Omega)$. Thus, (1.1) is equivalent to the linear problem

$$
\begin{gathered}
-\operatorname{div}\left[\alpha_{\epsilon}(x) \nabla U_{\epsilon}\right]=f \quad \text { in } \Omega, \\
U_{\epsilon}=0 \quad \text { on } \partial \Omega .
\end{gathered}
$$

that is, $u_{\epsilon}$ solves (1.1) is and only if $U_{\epsilon}$ solves (3.4).

From Lax-Milgram Lemma, there is at most one solution for (3.4), and therefore, there is also at most one solution for (1.1). Indeed, if there were two solutions for (1.1), we would be able to construct also two solutions for (3.4).

We now prove Theorem 3.2 .

Existence. Consider in Theorem 3.1 that $A=E=L^{2}(\Omega), C=H_{0}^{1}(\Omega)$, and the operator $T^{\epsilon}$ defined by (3.1). Then, from Lemma 3.3 we conclude that $T^{\epsilon}$ has a fixed point.

Uniqueness. Follows from Lemma 3.5.

To show existence of the RFB solution, it is enough to pursue the same ideas just presented, but now considering the operator

$$
T_{h}^{\epsilon}: L^{2}(\Omega) \rightarrow V_{r},
$$

where, for a given $w \in L^{2}(\Omega)$, we define $w_{r}^{\epsilon}=T_{h}^{\epsilon}(w)$ such that

$$
\int_{\Omega} \alpha_{\epsilon}(x) b(w) \nabla w_{r}^{\epsilon} . \nabla v d x=\int_{\Omega} f v d x \quad \text { for all } v \in V_{r} .
$$

As in Lemma 3.3, the operator $T_{h}^{\epsilon}$ is continuous. The proof is basically the same, replacing $H_{0}^{1}(\Omega)$ by $V_{r}$. 
Remark 3.6. In [26] the existence and uniqueness result for solutions for the MsFEM requires monotonicity. Such results were obtained [45] without monotonicity assumptions, but under the condition that the discrete and exact solutions are close. We follow the same approach.

To establish a uniqueness result, let $\mathcal{L} u=-\operatorname{div}\left[\alpha_{\epsilon}(x) b(u) \nabla u\right]$, and its Fréchet derivative in $u$ defined by

$$
\mathcal{L}^{\prime}(u) v=-\operatorname{div}\left\{\alpha_{\epsilon}(x) \nabla[b(u) v]\right\}=-\operatorname{div}\left\{\alpha_{\epsilon}(x)\left[b(u) \nabla v+b^{\prime}(u) v \nabla u\right] .\right.
$$

Consider also (1.5) and

$$
a^{\prime}(u ; v, \chi)=\int_{\Omega} \alpha_{\epsilon} \nabla[b(u) v] \cdot \nabla \chi=\int_{\Omega} \alpha_{\epsilon}\left[b(u) \nabla v \cdot \nabla \chi+b^{\prime}(u) v \nabla u \cdot \nabla \chi\right],
$$

induced by $\mathcal{L}$ and $\mathcal{L}^{\prime}$ respectively. From [39, Theorem 6 and Remark 6], it follows that $\mathcal{L}^{\prime}(u)$ defines an isomorphism from $H_{0}^{1}(\Omega)$ in $H^{-1}(\Omega)$. Note that if $\chi=b(u) v$, then

$$
\sup _{\chi \in H_{0}^{1}(\Omega)} \frac{a^{\prime}(u ; v, \chi)}{\|\chi\|_{1}} \geq \frac{\int_{\Omega} \alpha_{\epsilon}|\nabla[b(u) v]|^{2}}{\|b(u) v\|_{1}} \geq \alpha_{0}\|b(u) v\|_{1} \geq c(u)\|v\|_{1} .
$$

Note also that

$$
|b(u) v|_{1}=\left\|b(u) \nabla v+b^{\prime}(u) v \nabla u\right\|_{0} \geq\|b(u) \nabla v\|_{0}-\left\|b^{\prime}(u) v \nabla u\right\|_{0},
$$

and, on the other hand, from Poincaré's inequality,

$$
\left\|b^{\prime}(u) v \nabla u\right\|_{0} \leq\|\nabla b(u)\|_{L^{\infty}(\Omega)}\|v\|_{0} \leq C_{\Omega}\|\nabla b(u)\|_{L^{\infty}(\Omega)}\|\nabla v\|_{0} .
$$

It is enough to consider then

$$
c(u) \geq \alpha_{0}\left(b_{0}-C_{\Omega}\|\nabla b(u)\|_{L^{\infty}(\Omega)}\right) .
$$

Thus, for $\|u\|_{1, \infty}$ sufficiently small, $c(u)$ is positive.

In what follows, we consider the Galerkin projection $P_{h}: H_{0}^{1}(\Omega) \rightarrow V_{r}$ with respect to the bilinear form $\int_{\Omega} \alpha_{\epsilon}(x) b\left(u_{\epsilon}\right) \nabla v \nabla \chi d x$. Assume also that

$$
\left\|\chi-P_{h} \chi\right\|_{L^{2}(\Omega)} \leq \hat{c}(h)\|\chi\|_{H^{1}(\Omega)},
$$

where $\hat{c}(h) \rightarrow 0$ independently of $\epsilon$. This holds, for instance, if $\alpha(\cdot)$ is $\epsilon$-periodic [18].

Consider the following result.

Lemma 3.7. Let $u$ and $\tilde{u} \in H^{1}(\Omega)$. Then

$$
\bar{c}(u)\left\|v_{h}\right\|_{1} \leq \sup _{\chi_{h} \in V_{h}} \frac{a^{\prime}\left(\tilde{u} ; v_{h}, \chi_{h}\right)}{\left\|\chi_{h}\right\|_{1}}
$$

where $\bar{c}(u)=c(u)-\hat{c}(h)-\|u-\tilde{u}\|_{1, \infty}\|b\|_{2, \infty}\|\alpha\|_{0, \infty}\|u\|_{1, \infty}$. 
Proof. To show (3.5), note that

$$
\begin{gathered}
a^{\prime}\left(\tilde{u} ; v_{h}, \chi_{h}\right)=\int_{\Omega} \alpha_{\epsilon}\left[b(\tilde{u}) \nabla v_{h} \cdot \nabla \chi_{h}+b^{\prime}(\tilde{u}) v_{h} \nabla \tilde{u} \cdot \nabla \chi_{h}\right] \\
=\int_{\Omega} \alpha_{\epsilon}\left\{b(u) \nabla v_{h} \cdot \nabla \chi_{h}+b^{\prime}(u) v_{h} \nabla u \cdot \nabla \chi_{h}\right\} \\
+\int_{\Omega} \alpha_{\epsilon}\left\{[b(\tilde{u})-b(u)] \nabla v_{h} \cdot \nabla \chi_{h}+\left[b^{\prime}(\tilde{u}) \nabla \tilde{u}-b^{\prime}(u) \nabla u\right] v_{h} \cdot \nabla \chi_{h}\right\} \\
\geq a^{\prime}\left(u ; v_{h}, \chi_{h}\right)-\delta\left\|v_{h}\right\|_{1}\left\|\chi_{h}\right\|_{1}
\end{gathered}
$$

where

$$
\delta=\|\alpha\|_{0, \infty}\|b\|_{2, \infty}\|\tilde{u}\|_{1, \infty}\|\tilde{u}-u\|_{1, \infty} .
$$

Observe that, from [45, Lemma 2.2],

$$
a^{\prime}\left(u ; v_{h}, P_{h} \chi\right) \geq a^{\prime}\left(u ; v_{h}, \chi\right)-\hat{c}(h)\left\|v_{h}\right\|_{1}\|\chi\|_{1}
$$

for all $\chi \in H_{0}^{1}(\Omega)$. Then

$$
\begin{aligned}
& \sup _{\chi_{h} \in V_{r}} \frac{a^{\prime}\left(\tilde{u} ; v_{h}, \chi_{h}\right)}{\left\|\chi_{h}\right\|_{1}}=\sup _{\chi \in H_{0}^{1}(\Omega)} \frac{a^{\prime}\left(\tilde{u} ; v_{h}, P_{h} \chi\right)}{\left\|P_{h} \chi\right\|_{1}} \\
& \geq c \sup _{\chi \in H_{0}^{1}(\Omega)} \frac{a^{\prime}\left(u ; v_{h}, P_{h} \chi\right)}{\|\chi\|_{1}}-\delta\left\|v_{h}\right\|_{1} \geq \sup _{\chi \in H_{0}^{1}(\Omega)} \frac{a^{\prime}\left(u ; v_{h}, \chi\right)}{\|\chi\|_{1}}-(\hat{c}(h)+\delta)\left\|v_{h}\right\|_{1} \\
& \quad \geq[c(u)-\hat{c}(h)-\delta]\left\|v_{h}\right\|_{1} \geq \bar{c}(u)\left\|v_{h}\right\|_{1}
\end{aligned}
$$

for $\delta$ and $h$ sufficiently small. Above, we use the inequality $\left\|P_{h} \chi\right\|_{1} \leq c\|\chi\|_{1}$.

Theorem 3.8. Let $u_{h}$ and $\tilde{u}_{h}$ be two solutions for the discrete problem such that

$$
\left\|u-u_{h}\right\|_{1, \infty}+\left\|u-\tilde{u}_{h}\right\|_{1, \infty} \leq \eta
$$

where $\eta$ is small enough. Then $u_{h}=\tilde{u}_{h}$.

Proof. Note that

$$
\left\|u-u_{h}-t\left(\tilde{u}_{h}-u_{h}\right)\right\| \leq(1-t)\left\|u-u_{h}\right\|+t\left\|u-\tilde{u}_{h}\right\| \leq \eta,
$$

for all $t \in[0,1]$. Let $\eta$ be small enough such that

$$
\bar{c}(u)=c(u)-\hat{c}(h)-\eta\|b\|_{2, \infty}\|\alpha\|_{0, \infty}\|u\|_{1, \infty}>0 .
$$


Then

$$
\begin{aligned}
& \bar{c}(u)\left\|u_{h}-\tilde{u}_{h}\right\|_{1}=\bar{c}(u) \int_{0}^{1}\left\|u_{h}-\tilde{u}_{h}\right\|_{1} d t \\
& \leq \int_{0}^{1} \sup _{\chi_{h} \in V_{h}} \frac{a^{\prime}\left(u_{h}+t\left(\tilde{u}_{h}-u_{h}\right) ; u_{h}-\tilde{u}_{h}, \chi_{h}\right)}{\left\|\chi_{h}\right\|_{1}} d t \\
& \leq \sup _{\chi_{h} \in V_{h}} \frac{\int_{0}^{1} a^{\prime}\left(u_{h}+t\left(\tilde{u}_{h}-u_{h}\right) ; u_{h}-\tilde{u}_{h}, \chi_{h}\right) d t}{\left\|\chi_{h}\right\|_{1}} \\
&=\sup _{\chi_{h} \in V_{h}} \frac{\int_{0}^{1} \frac{d}{d t} a\left(u_{h}+t\left(\tilde{u}_{h}-u_{h}\right), \chi_{h}\right) d t}{\left\|\chi_{h}\right\|_{1}}=0 .
\end{aligned}
$$

Since $\bar{c}(u)>0$, then $u_{h}=\tilde{u}_{h}$.

\section{Best APPRoximation REsult}

We establish here a Céa's Lemma type result for the Residual Free Bubble Method. The strategy to obtain such result is to consider a linearization $A\left(u_{r} ; \cdot, \cdot\right)$ of (1.5) centered at the "enriched solution" $u_{r}$. We consider then the following linear problem to find $w \in H_{0}^{1}(\Omega)$ such that

$$
A\left(u_{r} ; w, v\right)=(f, v) \quad \text { for all } v \in H_{0}^{1}(\Omega)
$$

where

$$
A\left(u_{r} ; w, v\right)=\int_{\Omega} \alpha_{\epsilon}(x) b\left(u_{r}\right) \nabla w \cdot \nabla v d x
$$

Thus, $A\left(u_{r} ; \cdot, \cdot\right)$ is coercive in $H_{0}^{1}(\Omega)$, since

$$
A\left(u_{r} ; w, w\right)=\int_{\Omega} \alpha_{\epsilon}(x) b\left(u_{r}\right)|\nabla w|^{2} d x \geq C_{\Omega} \alpha_{0} b_{0}\|w\|_{1, \Omega}^{2}
$$

where $C_{\Omega}$ is the Poincaré's constant.

We establish first the following identity.

Lemma 4.1. Given $v_{r} \in V_{r}$, the following identity holds

$$
A\left(u_{r} ; u_{\epsilon}-u_{r}, v_{r}\right)=A\left(u_{\epsilon} ; u^{\epsilon}-u_{r}, v_{r}\right)=\int_{\Omega} \alpha_{\epsilon}(x)\left[b\left(u_{r}\right)-b\left(u_{\epsilon}\right)\right] \nabla u_{\epsilon} \cdot \nabla v_{r} d x
$$


Proof. Indeed,

$$
\begin{gathered}
A\left(u_{r} ; u_{\epsilon}-u_{r}, v_{r}\right)=\int_{\Omega} \alpha_{\epsilon}(x) b\left(u_{r}\right) \nabla u_{\epsilon} \cdot \nabla v_{r} d x-\int_{\Omega} \alpha_{\epsilon}(x) b\left(u_{r}\right) \nabla u_{r} \cdot \nabla v_{r} d x \\
=\int_{\Omega} \alpha_{\epsilon}(x) b\left(u_{r}\right) \nabla u_{\epsilon} \cdot \nabla v_{r} d x-\int_{\Omega} f v_{r} d x \\
=\int_{\Omega} \alpha_{\epsilon}(x) b\left(u_{r}\right) \nabla u_{\epsilon} \cdot \nabla v_{r} d x-\int_{\Omega} \alpha_{\epsilon}(x) b\left(u_{\epsilon}\right) \nabla u_{\epsilon} \cdot \nabla v_{r} d x \\
=\int_{\Omega} \alpha_{\epsilon}(x)\left[b\left(u_{r}\right)-b\left(u^{\epsilon}\right)\right] \nabla u^{\epsilon} \cdot \nabla v_{r} d x .
\end{gathered}
$$

The proof of the second inequality is similar.

We end the present section establishing a best approximation result in the enriched space $V_{r}$. This is a Céa's Lemma type result for the multiscale nonlinear problem [10]. An advantage of the estimate is that it requires less regularity of $b(\cdot)$ than in [20], cf. also Remark 4.4.

We often use Hölder's inequality

$$
\int_{\Omega} f g h d x \leq\|f\|_{L^{3}}\|g\|_{L^{6}}\|h\|_{L^{2}} \leq\|f\|_{0, \Omega}^{1 / 2}\|f\|_{1, \Omega}^{1 / 2}\|g\|_{L^{6}}\|h\|_{L^{2}}
$$

where we use also the continuous embedding $H^{1}(\Omega) \hookrightarrow L^{6}(\Omega)$ (for dimensions smaller than three).

Proposition 4.2. Let $\alpha_{\epsilon}($.$) and b($.$) satisfying (1.2) and (1.3), respectively. Then, for u_{\epsilon}$ sufficiently small in $W^{1,6}(\Omega)$, it follows that

$$
\left\|\nabla\left(u_{\epsilon}-u_{r}\right)\right\|_{0, \Omega} \leq C\left\|\nabla\left(u_{\epsilon}-w_{r}\right)\right\|_{0, \Omega} \quad \text { for all } w_{r} \in V_{r} .
$$

Proof. Let $w_{r} \in V_{r}$. To establish (4.3), compute

$$
\begin{aligned}
& A\left(u_{r} ; u_{\epsilon}-u_{r}, u_{\epsilon}-u_{r}\right)=A\left(u_{r} ; u_{\epsilon}-u_{r}, u_{\epsilon}-w_{r}\right)+A\left(u_{r} ; u_{\epsilon}-u_{r}, w_{r}-u_{r}\right) \\
& =\int_{\Omega} \alpha_{\epsilon} b\left(u_{r}\right) \nabla\left(u^{\epsilon}-u_{r}\right) \cdot \nabla\left(u^{\epsilon}-w_{r}\right) d x+\int_{\Omega} \alpha_{\epsilon}\left(b\left(u_{r}\right)-b\left(u_{\epsilon}\right)\right) \nabla u^{\epsilon} \cdot \nabla\left(w_{r}-u_{r}\right) d x
\end{aligned}
$$

using (4.2). Denote by $I_{1}, I_{2}$ the first and second terms of (4.4). We now estimate each of these terms

$$
I_{1}=\int_{\Omega} \alpha_{\epsilon} b\left(u_{r}\right) \nabla\left(u^{\epsilon}-u_{r}\right) \cdot \nabla\left(u_{\epsilon}-w_{r}\right) d x \leq c_{1}\left\|\nabla\left(u_{\epsilon}-u_{r}\right)\right\|_{0, \Omega}\left\|\nabla\left(u_{\epsilon}-w_{r}\right)\right\|_{0, \Omega},
$$


where $c_{1}:=\alpha_{1}\|b\|_{\infty}$. We estimate now $I_{2}$ :

$$
\begin{gathered}
I_{2}=\int_{\Omega} \alpha_{\epsilon}\left(b\left(u_{r}\right)-b\left(u_{\epsilon}\right)\right) \nabla u_{\epsilon} \cdot \nabla\left(w_{r}-u_{r}\right) d x \\
\quad \leq \alpha_{1}\left\|b^{\prime}\right\|_{\infty} \int_{\Omega}\left|u_{r}-u_{\epsilon}\right|\left|\nabla u_{\epsilon}\right|\left|\nabla\left(w_{r}-u_{r}\right)\right| d x \\
\leq \alpha_{1}\left\|b^{\prime}\right\|_{\infty}\left\|u_{r}-u_{\epsilon}\right\|_{L^{2}(\Omega)}^{1 / 2}\left\|u_{r}-u_{\epsilon}\right\|_{H^{1}(\Omega)}^{1 / 2}\left\|\nabla u_{\epsilon}\right\|_{L^{6}(\Omega)}\left\|w_{r}-u_{r}\right\|_{1, \Omega} \\
\leq \alpha_{1}\left\|b^{\prime}\right\|_{\infty}\left\|\nabla u_{\epsilon}\right\|_{L^{6}(\Omega)}\left\|u_{\epsilon}-u_{r}\right\|_{H^{1}(\Omega)}\left[\left\|u_{\epsilon}-u_{r}\right\|_{1, \Omega}+\left\|u_{\epsilon}-w_{r}\right\|_{1, \Omega}\right] .
\end{gathered}
$$

From (4.1), there exists $\beta>0$, independent of $\epsilon$, such that

$$
A\left(u_{r} ; u_{\epsilon}-u_{r}, u_{\epsilon}-u_{r}\right) \geq \beta\left\|\nabla\left(u^{\epsilon}-u_{r}\right)\right\|_{0, \Omega}^{2} .
$$

Moreover, from the estimates for $I_{1}, I_{2}$ in (4.4), we gather that

$$
\begin{aligned}
\beta\left\|\nabla\left(u_{\epsilon}-u_{r}\right)\right\|_{0, \Omega}^{2} \leq & c_{1}\left\|\nabla\left(u_{\epsilon}-u_{r}\right)\right\|_{0, \Omega}\left\|\nabla\left(u_{\epsilon}-w_{r}\right)\right\|_{0, \Omega} \\
& +\alpha_{1}\left\|b^{\prime}\right\|_{\infty}\left\|\nabla u_{\epsilon}\right\|_{L^{6}(\Omega)}\left\|u_{\epsilon}-u_{r}\right\|_{H^{1}(\Omega)}\left[\left\|u_{\epsilon}-u_{r}\right\|_{1, \Omega}+\left\|u_{\epsilon}-w_{r}\right\|_{1, \Omega}\right] .
\end{aligned}
$$

Thus

$$
\begin{aligned}
\beta\left\|\nabla\left(u_{\epsilon}-u_{r}\right)\right\|_{0, \Omega} \leq\left(c_{1}+\alpha_{1}\left\|b^{\prime}\right\|_{\infty}\left\|\nabla u_{\epsilon}\right\|_{L^{6}(\Omega)}\right) \| u_{\epsilon}- & w_{r} \|_{H^{1}(\Omega)} \\
& +\alpha_{1}\left\|b^{\prime}\right\|_{\infty}\left\|\nabla u_{\epsilon}\right\|_{L^{6}(\Omega)}\left\|u_{\epsilon}-u_{r}\right\|_{1, \Omega},
\end{aligned}
$$

and then

$$
\left\|u_{\epsilon}-u_{r}\right\|_{1, \Omega}\left(\beta-\alpha_{1}\left\|b^{\prime}\right\|_{\infty}\left\|\nabla u_{\epsilon}\right\|_{L^{6}(\Omega)}\right) \leq c_{1}\left\|u_{\epsilon}-w_{r}\right\|_{1, \Omega}
$$

Remark 4.3. Proposition 4.2 is important because the best approximation estimate is independent of $\epsilon$, and shows in particular that the RFB method converges at least as well as the MsFEM since the RFB approximation spaces contains the spaces employed in the MsFEM. The choice of the approximation spaces is crucial here, since polynomial spaces with no bubbles added, a.k.a. classical Galerkin, yield a method that converges in h albeit non-uniformly with respect to $\epsilon$.

Remark 4.4. Dropping the "small solution" hypothesis, (also present in [1]), an analogous result holds. In particular, the estimate

$$
\left\|u_{\epsilon}-u_{r}\right\|_{H^{1}(\Omega)} \leq C\left(\left\|u_{\epsilon}-w_{r}\right\|_{H^{1}(\Omega)}+\left\|u_{\epsilon}-u_{r}\right\|_{L^{2}(\Omega)}\right) \quad \text { for all } w_{r} \in V_{r}
$$


results from the above proof. An estimate for $\left\|u_{\epsilon}-u_{r}\right\|_{H^{1}(\Omega)}$ was obtained in [20, Theorem 1], under extra regularity for $b(\cdot)$. Following their proof, it is possible to show that

$$
\begin{array}{r}
\left\|u_{\epsilon}-u_{r}\right\|_{H^{1}(\Omega)} \leq C\left\|u_{\epsilon}-w_{r}\right\|_{H^{1}(\Omega)}\left(1+\inf _{\tilde{\chi} \in V_{r}}\|\phi-\tilde{\chi}\|_{H^{1}(\Omega)}+\left\|u_{\epsilon}-w_{r}\right\|_{H^{1}(\Omega)}^{2}\right) \\
+C\left\|u_{\epsilon}-u_{r}\right\|_{L^{2}(\Omega)}\left(\inf _{\tilde{\chi} \in V_{r}}\|\phi-\tilde{\chi}\|_{H^{1}(\Omega)}+\left\|u_{\epsilon}-u_{r}\right\|_{L^{2}(\Omega)}^{2}\right),
\end{array}
$$

for all $w_{r} \in V_{r}$, where $\phi$ is the solution of a linear dual problem. It follows then that $\| u_{\epsilon}-$ $u_{r} \|_{L^{2}(\Omega)}$ is small enough as long as the mesh size $h$ is small enough, and a best approximation result follows. However, the compactness argument of [20] does not allow, in principle, the mesh size to be independent of the small scales.

Finally, strict monotonicity is also a sufficient condition for the best approximation result of Lemma [27], i.e,

$$
\int_{\Omega} \alpha_{\epsilon}(x)\left[b\left(v_{r}\right) \nabla v_{r}-b\left(w_{r}\right) \nabla w_{r}\right] \cdot \nabla\left(v_{r}-w_{r}\right) d x \geq c\left\|v_{r}-w_{r}\right\|_{H^{1}(\Omega)}^{2}
$$

for all $v_{r}, w_{r} \in V_{r}$. In this case,

$$
\begin{aligned}
&\left\|u_{r}-w_{r}\right\|_{H^{1}(\Omega)}^{2} \leq c \int_{\Omega} \alpha_{\epsilon}(x)\left[b\left(u_{r}\right) \nabla u_{r}-b\left(w_{r}\right) \nabla w_{r}\right] \cdot \nabla\left(u_{r}-w_{r}\right) d x \\
&= c \int_{\Omega} \alpha_{\epsilon}(x)\left[b\left(u_{\epsilon}\right) \nabla u_{\epsilon}-b\left(w_{r}\right) \nabla w_{r}\right] \cdot \nabla\left(u_{r}-w_{r}\right) d x \\
& \leq c\left\|b\left(u_{\epsilon}\right) \nabla u_{\epsilon}-b\left(w_{r}\right) \nabla w_{r}\right\|_{L^{2}(\Omega)}\left\|u_{r}-w_{r}\right\|_{H^{1}(\Omega)} \\
& \leq c\left(\left\|b\left(u_{\epsilon}\right) \nabla u_{\epsilon}-b\left(w_{r}\right) \nabla u_{\epsilon}\right\|_{L^{2}(\Omega)}+\left\|b\left(w_{r}\right) \nabla u_{\epsilon}-b\left(w_{r}\right) \nabla w_{r}\right\|_{L^{2}(\Omega)}\right)\left\|u_{r}-w_{r}\right\|_{H^{1}(\Omega)},
\end{aligned}
$$

and we conclude that $\left\|u_{r}-w_{r}\right\|_{H^{1}(\Omega)} \leq c\left\|u_{\epsilon}-w_{r}\right\|_{H^{1}(\Omega)}$ for all $w_{r} \in V_{r}$. An estimate as (4.3) follows from the triangle inequality.

\section{Possible Linearizations}

As in the original problem (1.1), the RFB approximation (2.2), or equivalently (2.1), is still given by a nonlinear problem. We investigate here some ideas to linearize the problem. In the next subsection, we investigate fixed point schemes, and in the following subsection, we discuss a proposal named reduced $R F B$.

5.1. Fixed point formulation. A first idea to linearize the original problem (1.1) is the following. Let $u_{\epsilon}^{0} \in H_{0}^{1}(\Omega)$, and for $n \in \mathbb{N}$, given $u_{\epsilon}^{n-1} \in H_{0}^{1}(\Omega)$, compute $u_{\epsilon}^{n} \in H_{0}^{1}(\Omega)$ as the solution of

$$
\int_{\Omega} \alpha_{\epsilon}(x) b\left(u_{\epsilon}^{n-1}\right) \nabla\left(u_{\epsilon}^{n}\right) \cdot \nabla v d x=\int_{\Omega} f v d x \quad \text { for all } v \in H_{0}^{1}(\Omega)
$$


In the context of the RFB method, we use (2.1) to propose the following iterative scheme. Let $u_{\epsilon}^{0} \in V_{r}$, and $n \in \mathbb{N}$. Given $u_{r}^{n-1} \in V_{r}$, compute $u_{r}^{n} \in V_{r}$ solution of

$$
\int_{\Omega} \alpha_{\epsilon}(x) b\left(u_{r}^{n-1}\right) \nabla\left(u_{r}^{n}\right) \cdot \nabla v_{r} d x=\int_{\Omega} f v_{r} d x \quad \text { for all } v_{r} \in V_{r} .
$$

Observe that the above scheme discretizes (5.1). Hence, discretization and linearization commutes. Since the problem now is linear, we head back to the situation described in Remark 2.1.

We can also rewrite (5.2) in terms of global/local problems. Given $u_{h}^{n-1} \in V_{h}$ and $u_{b}^{n-1} \in$ $V_{b}$, find $u_{h}^{n} \in V_{h}$ and $u_{b}^{n} \in V_{b}$ such that

$$
\begin{gathered}
\int_{\Omega} \alpha_{\epsilon}(x) b\left(u_{h}^{n-1}+u_{b}^{n-1}\right) \nabla\left(u_{h}^{n}+u_{b}^{n}\right) \cdot \nabla v_{h} d x=\int_{\Omega} f v_{h} d x, \\
-\operatorname{div}\left[\alpha_{\epsilon}(x) b\left(u_{h}^{n-1}+u_{b}^{n-1}\right) \nabla\left(u_{h}^{n}+u_{b}^{n}\right)\right]=f \quad \text { in } K
\end{gathered}
$$

for all $v_{h} \in V_{h}$ and all $K \in \mathcal{T}_{h}$.

Lemma 5.1. Given $u_{\epsilon}^{0} \in H_{0}^{1}(\Omega)$ and $u_{r}^{0} \in V_{r}$, let $u_{\epsilon}^{n} \in H_{0}^{1}(\Omega)$ and $u_{r}^{n} \in V_{r}$ be defined from (5.1) and (5.2) for $n \in \mathbb{N}$. Then $\lim _{n \rightarrow \infty} u_{\epsilon}^{n}=u_{\epsilon}$ and $\lim _{n \rightarrow \infty} u_{r}^{n}=u_{r}$ in $H_{0}^{1}(\Omega)$.

Proof. We first consider the continuous problem, for a fixed $\epsilon$. Note that $\left\|\nabla u_{\epsilon}^{n}\right\|_{0} \leq c\|f\|_{-1}$, and then $\left\|\nabla u_{\epsilon}^{n}\right\|_{0, \Omega}$ is bounded. Therefore, there exist $\bar{u} \in H_{0}^{1}(\Omega)$ and a subsequence of $u_{\epsilon}^{n}$, indexed by $n \in \mathbb{N}$, but still denoted by $u_{\epsilon}^{n}$, such that $u_{\epsilon}^{n}$ weakly converges to $\bar{u}$ in $H_{0}^{1}(\Omega)$, with strong convergence in $L^{2}(\Omega)$. Thus, from the Lebesgue Dominated Convergence Theorem, $b\left(u_{\epsilon}^{n}\right) \nabla v \rightarrow b(\bar{u}) \nabla v$ strongly in $L^{2}(\Omega)$, for all $v \in H_{0}^{1}(\Omega)$. Note also that $\int_{\Omega} \nabla\left(u_{\epsilon}^{n}-\bar{u}\right)$. $\boldsymbol{\tau} d x \rightarrow 0$ for all $\boldsymbol{\tau} \in L^{2}(\Omega)$. Indeed, from Helmholtz decomposition, there exist $p \in H_{0}^{1}(\Omega)$, $q \in H^{1}(\Omega)$ such that $\boldsymbol{\tau}=\nabla p+\operatorname{curl} q$. Therefore,

$$
\int_{\Omega} \nabla\left(u_{\epsilon}^{n}-\bar{u}\right) \cdot \tau d x=\int_{\Omega} \nabla\left(u_{\epsilon}^{n}-\bar{u}\right) \cdot \nabla p d x \rightarrow 0
$$

as $n \rightarrow \infty$. It follows from these results that, for all $v \in H_{0}^{1}(\Omega)$,

$$
\begin{aligned}
& \int_{\Omega}\left[b\left(u_{\epsilon}^{n-1}\right) \nabla u_{\epsilon}^{n}\right.-b(\bar{u}) \nabla \bar{u}] \nabla v \\
&= \int_{\Omega}\left[b\left(u_{\epsilon}^{n-1}\right)-b(\bar{u})\right] \nabla u_{\epsilon}^{n} \nabla v+\int_{\Omega} b(\bar{u})\left[\nabla u_{\epsilon}^{n}-\nabla \bar{u}\right] \nabla v \\
& \leq\left\|\left[b\left(u_{\epsilon}^{n-1}\right)-b(\bar{u})\right] \nabla v\right\|_{0}\left\|\nabla u_{\epsilon}^{n}\right\|_{0}+\int_{\Omega} b(\bar{u})\left[\nabla u_{\epsilon}^{n}-\nabla \bar{u}\right] \nabla v .
\end{aligned}
$$

Taking $n \rightarrow \infty$ we gather that

$$
\int_{\Omega}\left[b\left(u_{\epsilon}^{n-1}\right) \nabla u_{\epsilon}^{n}-b(\bar{u}) \nabla \bar{u}\right] \nabla v \rightarrow 0 .
$$


Thus

$$
0=\lim _{n \rightarrow \infty} \int_{\Omega} b\left(u_{\epsilon}^{n-1}\right) \nabla u_{\epsilon}^{n} \nabla v-f v d x=\int_{\Omega} b(\bar{u}) \nabla \bar{u} \nabla v-f v d x .
$$

Then $\bar{u}$ solves (1.1). From uniqueness of solutions, $\bar{u}=u_{\epsilon}$, and the whole sequence, and not only a subsequence, $u_{\epsilon}^{n}$ converges to $\bar{u}$.

To show that the convergence is actually strong, note [16] that

$$
\begin{gathered}
\left\|u_{\epsilon}^{n}-\bar{u}\right\|_{H^{1}(\Omega)} \leq \int_{\Omega} \alpha_{\epsilon} b\left(u_{\epsilon}^{n-1}\right) \nabla\left(u_{\epsilon}^{n}-\bar{u}\right) \cdot \nabla\left(u_{\epsilon}^{n}-\bar{u}\right) d x \\
=\int_{\Omega} \alpha_{\epsilon} b\left(u_{\epsilon}^{n-1}\right) \nabla(\bar{u}) \cdot \nabla\left(\bar{u}-2 u_{\epsilon}^{n}\right) d x+\int_{\Omega} \alpha_{\epsilon} b\left(u_{\epsilon}^{n-1}\right) \nabla\left(u_{\epsilon}^{n}\right) \cdot \nabla\left(u_{\epsilon}^{n}\right) d x \\
=\int_{\Omega} \alpha_{\epsilon} b\left(u_{\epsilon}^{n-1}\right) \nabla(\bar{u}) \cdot \nabla\left(\bar{u}-2 u_{\epsilon}^{n}\right) d x+\int_{\Omega} f u_{\epsilon}^{n} d x \\
\rightarrow-\int_{\Omega} \alpha_{\epsilon} b(\bar{u}) \nabla(\bar{u}) \cdot \nabla(\bar{u}) d x+\int_{\Omega} f \bar{u} d x
\end{gathered}
$$

since (5.4) holds. Thus the convergence $u_{\epsilon}^{n} \rightarrow \bar{u}$ is strong in $H^{1}(\Omega)$.

The second part of the lemma, regarding the RFB approximation, follows from basically the same arguments. Since $\left\|\nabla u_{r}^{n}\right\|_{0} \leq c\|f\|_{-1}$, there exists $\bar{u}_{r} \in H_{0}^{1}(\Omega)$ and a subsequence still denoted by $u_{r}^{n}$ such that $u_{r}^{n}$ weakly converges to $\bar{u}_{r}$ in $H_{0}^{1}(\Omega)$, whereas strong convergence holds in $L^{2}(\Omega)$. Again, $b\left(u_{r}^{n}\right) \nabla v \rightarrow b\left(\bar{u}_{r}\right) \nabla v$ strongly in $L^{2}(\Omega)$, for all $v \in H_{0}^{1}(\Omega)$. Note also that $\int_{\Omega} \nabla\left(u_{r}^{n}-\bar{u}_{r}\right) \cdot \tau d x \rightarrow 0$ for all $\tau \in L^{2}(\Omega)$. Indeed, from Helmholtz decomposition, there exist $p \in H_{0}^{1}(\Omega), q \in H^{1}(\Omega)$ such that $\boldsymbol{\tau}=\nabla p+\operatorname{curl} q$. Thus

$$
\int_{\Omega} \nabla\left(u_{r}^{n}-\bar{u}_{r}\right) \cdot \tau d x=\int_{\Omega} \nabla\left(u_{r}^{n}-\bar{u}_{r}\right) \cdot \nabla p d x \rightarrow 0
$$

as $n \rightarrow \infty$. From these results, we gather that for all $v \in H_{0}^{1}(\Omega)$,

$$
\begin{aligned}
& \int_{\Omega}\left[b\left(u_{r}^{n-1}\right) \nabla u_{r}^{n}-b\left(\bar{u}_{r}\right) \nabla \bar{u}_{r}\right] \nabla v \\
&=\int_{\Omega}\left[b\left(u_{r}^{n-1}\right)-b\left(\bar{u}_{r}\right)\right] \nabla u_{r}^{n} \nabla v+\int_{\Omega} b\left(\bar{u}_{r}\right)\left[\nabla u_{r}^{n}-\nabla \bar{u}_{r}\right] \nabla v \\
& \leq\left\|\left[b\left(u_{r}^{n-1}\right)-b\left(\bar{u}_{r}\right)\right] \nabla v\right\|_{0}\left\|\nabla u_{r}^{n}\right\|_{0}+\int_{\Omega} b\left(\bar{u}_{r}\right)\left[\nabla u_{r}^{n}-\nabla \bar{u}_{r}\right] \nabla v .
\end{aligned}
$$

Taking $n \rightarrow \infty$, it follows that $\int_{\Omega}\left[b\left(u_{r}^{n}\right) \nabla u_{r}^{n}-b\left(\bar{u}_{r}\right) \nabla \bar{u}_{r}\right] \nabla v \rightarrow 0$ for all $v \in H_{0}^{1}(\Omega)$. Considering now $v \in V_{r}$, we have that

$$
0=\lim _{n \rightarrow \infty} \int_{\Omega} b\left(u_{r}^{n-1}\right) \nabla u_{r}^{n} \nabla v-f v d x=\int_{\Omega} b\left(\bar{u}_{r}\right) \nabla \bar{u}_{r} \nabla v-f v d x .
$$

Since $V_{r}$ is closed, $\bar{u}_{r} \in V_{r}$. Therefore $\bar{u}_{r}=u_{r}$ solves (2.1). If uniqueness also holds, the whole sequence $u_{\epsilon}^{n}$ converges to $\bar{u}$. 
Lemma 5.2. Given $u_{\epsilon}^{0} \in H_{0}^{1}(\Omega)$ and $u_{r}^{0} \in V_{r}$, let $u_{\epsilon}^{n} \in H_{0}^{1}(\Omega)$ and $u_{r}^{n} \in V_{r}$ be defined by (5.1) and (5.2), $n \in \mathbb{N}$. Then, if $u_{\epsilon}$ is sufficiently small in $W^{1,6}(\Omega)$, we have that

$$
\left\|u_{\epsilon}^{n}-u_{\epsilon}\right\|_{H^{1}(\Omega)}+\left\|u_{r}^{n}-u_{r}\right\|_{H^{1}(\Omega)} \leq \bar{\alpha}\left\|u_{\epsilon}^{n-1}-u_{\epsilon}\right\|_{H^{1}(\Omega)},
$$

for $\bar{\alpha}<1$.

Proof. Note that

$$
\begin{aligned}
&\left\|u_{\epsilon}^{n}-u_{\epsilon}\right\|_{H^{1}(\Omega)}^{2} \leq \int_{\Omega} \alpha_{\epsilon}(x) b\left(u_{\epsilon}^{n-1}\right) \nabla\left(u_{\epsilon}^{n}-u_{\epsilon}\right) \nabla\left(u_{\epsilon}^{n}-u_{\epsilon}\right) d x \\
&= \int_{\Omega} \alpha_{\epsilon}(x)\left[-b\left(u_{\epsilon}^{n-1}\right)+b\left(u_{\epsilon}\right)\right] \nabla u_{\epsilon} \nabla\left(u_{\epsilon}^{n}-u_{\epsilon}\right) d x \\
& \quad \leq c\left\|u_{\epsilon}^{n-1}-u_{\epsilon}\right\|_{L^{2}(\Omega)}^{1 / 2}\left\|u_{\epsilon}^{n-1}-u_{\epsilon}\right\|_{H^{1}(\Omega)}^{1 / 2}\left\|\nabla u_{\epsilon}\right\|_{L^{6}(\Omega)}\left\|u_{\epsilon}^{n}-u_{\epsilon}\right\|_{H^{1}(\Omega)} .
\end{aligned}
$$

The result for $u_{r}^{n}$ is analogous.

We end this subsection with an alternative linearization proposal, based on (5.3). Given $u_{h}^{n-1} \in V_{h}$ and $u_{b}^{n-1} \in V_{b}$, find $u_{h}^{n} \in V_{h}$ and $u_{b}^{n} \in V_{b}$ such that

$$
\begin{gathered}
\int_{\Omega} \alpha_{\epsilon}(x) b\left(u_{h}^{n-1}+u_{b}^{n-1}\right) \nabla\left(u_{h}^{n}+u_{b}^{n-1}\right) \cdot \nabla v_{h} d x=\int_{\Omega} f v_{h} d x, \\
-\operatorname{div}\left[\alpha_{\epsilon}(x) b\left(u_{h}^{n}+u_{b}^{n-1}\right) \nabla\left(u_{h}^{n}+u_{b}^{n}\right)\right]=f \quad \text { in } K,
\end{gathered}
$$

for all $v_{h} \in V_{h}$ and all $K \in \mathcal{T}_{h}$. Observe that the above system is not coupled as in (5.3). It is possible to solve first (5.5) and only then solve (5.6).

5.2. Reduced Residual Free Bubble Formulation. The idea here is to use the approximation $b\left(u_{h}+u_{b}\right) \approx b\left(u_{h}\right)$ at the local problem of the second equation in (2.2). This induces a linearization that makes static condensation possible. In this case, we search for the approximation $\tilde{u}_{r}=\tilde{u}_{h}+\tilde{u}_{b} \in V_{r}$ such that

$$
\begin{gathered}
\int_{\Omega} \alpha_{\epsilon}(x) b\left(\tilde{u}_{h}+\tilde{u}_{b}\right) \nabla\left(\tilde{u}_{h}+\tilde{u}_{b}\right) \cdot \nabla v_{h} d x=\int_{\Omega} f v_{h} d x, \\
-\operatorname{div}\left[\alpha_{\epsilon}(x) b\left(\tilde{u}_{h}\right) \nabla \tilde{u}_{b}\right]=f+\operatorname{div}\left[\alpha_{\epsilon}(x) b\left(\tilde{u}_{h}\right) \nabla \tilde{u}_{h}\right] \quad \text { in } K,
\end{gathered}
$$

for all $v_{h} \in V_{h}$ and all $K \in \mathcal{T}_{h}$. Thus, the local problem (5.7) is linear with respect to $\tilde{u}_{b}$.

Remark 5.3. Since (5.7) is linear, we can split $\tilde{u}_{b}=\tilde{u}_{b}^{l}+\tilde{u}_{b}^{f}$ in two parts, each solving (5.7) with $f$ and $\operatorname{div}\left[\alpha_{\epsilon}(x) b\left(\tilde{u}_{h}\right) \nabla \tilde{u}_{h}\right]$ on the right hand side. However, the local and global problems are still coupled. The local problems for the MsFEM involve $\tilde{u}_{b}^{l}$ only, and to make the method cheaper, it is possible to replace $b\left(u_{h}\right)$ by $b\left(\int_{K} u_{h}(x) d x\right)$, as in [24], or by $\left(b\left(u_{h}\left(x_{K}\right)\right)\right)$ as in [18], where $x_{K}$ is an interior point of the element. In this way, (5.7) reduces to a much simpler equation, given by

$$
-\operatorname{div}\left[\alpha_{\epsilon}(x) \nabla \tilde{u}_{b}^{l}\right]=\operatorname{div}\left[\alpha_{\epsilon}(x) \nabla \tilde{u}_{h}\right] \quad \text { in } K .
$$


From the equation linearity, the computation of the local bubble $u_{b}^{l}$ is determined solving the corresponding problems associated to the basis functions.

However, such simplification is not possible for the RFB method, due to the presence of the $\tilde{u}_{b}^{f}$ term. Such extra term is important since it can significantly improve the quality of the approximation [29, 30, 41] in some situations.

\section{REFERENCES}

[1] Assyr Abdulle and Gilles Vilmart, A priori error estimates for finite element methods with numerical quadrature for nonmonotone nonlinear elliptic problems, Numer. Math. 121 (2012), no. 3, 397-431, DOI 10.1007/s00211-011-0438-4. MR2929073

[2] Rodolfo Araya, Christopher Harder, Diego Paredes, and Frédéric Valentin, Multiscale hybrid-mixed method, SIAM J. Numer. Anal. 51 (2013), no. 6, 3505-3531, DOI 10.1137/120888223. MR3143841

[3] J.-L. Auriault and J. Lewandowska, Homogenization analysis of diffusion and adsorption macrotransport in porous media: macrotransport in the absence of advection, Géotechnique 43 (01 September 1993), no. 3, 457-469.

[4] Michel Artola and Georges Duvaut, Un résultat d'homogénéisation pour une classe de problèmes de diffusion non linéaires stationnaires, Ann. Fac. Sci. Toulouse Math. (5) 4 (1982), no. 1, 1-28 (French, with English summary). MR673637 (84j:35020)

[5] Grégoire Allaire, Homogenization and two-scale convergence, SIAM J. Math. Anal. 23 (1992), no. 6, 1482-1518, DOI 10.1137/0523084. MR1185639 (93k:35022)

[6] Ivo Babuška, Gabriel Caloz, and John E. Osborn, Special finite element methods for a class of second order elliptic problems with rough coefficients, SIAM J. Numer. Anal. 31 (1994), no. 4, 945-981, DOI 10.1137/0731051. MR1286212 (95g:65146)

[7] I. Babuška and J. E. Osborn, Generalized finite element methods: their performance and their relation to mixed methods, SIAM J. Numer. Anal. 20 (1983), no. 3, 510-536, DOI 10.1137/0720034. MR701094 (84h:65076)

[8] Claudio Baiocchi, Franco Brezzi, and Leopoldo P. Franca, Virtual bubbles and Galerkin-least-squares type methods (Ga.L.S.), Comput. Methods Appl. Mech. Engrg. 105 (1993), no. 1, 125-141, DOI 10.1016/0045-7825(93)90119-I. MR1222297 (94g:65058)

[9] Alain Bensoussan, Jacques-Louis Lions, and George Papanicolaou, Asymptotic analysis for periodic structures, Studies in Mathematics and its Applications, vol. 5, North-Holland Publishing Co., Amsterdam, 1978. MR503330 (82h:35001)

[10] Susanne C. Brenner and L. Ridgway Scott, The mathematical theory of finite element methods, 3rd ed., Texts in Applied Mathematics, vol. 15, Springer, New York, 2008. MR2373954 (2008m:65001)

[11] Lucio Boccardo and François Murat, Remarques sur l'homogénéisation de certains problèmes quasilinéaires, Portugal. Math. 41 (1982), no. 1-4, 535-562 (1984) (French, with English summary). MR766874 (86a:35022)

[12] Haim Brezis, Functional analysis, Sobolev spaces and partial differential equations, Universitext, Springer, New York, 2011. MR2759829 (2012a:35002)

[13] F. Brezzi, Interacting with the subgrid world, Numerical analysis 1999 (Dundee), Chapman \& Hall/CRC Res. Notes Math., vol. 420, Chapman \& Hall/CRC, Boca Raton, FL, 2000, pp. 69-82. MR1751112 (2001b:65121)

[14] Franco Brezzi, Marie Odile Bristeau, Leopoldo P. Franca, Michel Mallet, and Gilbert Rogé, A relationship between stabilized finite element methods and the Galerkin method with bubble functions, Comput. Methods Appl. Mech. Engrg. 96 (1992), no. 1, 117-129, DOI 10.1016/0045-7825(92)90102-P. MR1159592 (92k:76056) 
[15] Franco Brezzi and Alessandro Russo, Choosing bubbles for advection-diffusion problems, Math. Models Methods Appl. Sci. 4 (1994), no. 4, 571-587, DOI 10.1142/S0218202594000327. MR1291139 (95h:76079)

[16] Philippe G. Ciarlet, Mathematical elasticity. Vol. II, Studies in Mathematics and its Applications, vol. 27, North-Holland Publishing Co., Amsterdam, 1997. Theory of plates. MR1477663 (99e:73001)

[17] Zhangxin Chen, Multiscale methods for elliptic homogenization problems, Numer. Methods Partial Differential Equations 22 (2006), no. 2, 317-360, DOI 10.1002/num.20099. MR2201437 (2007b:65117)

[18] Zhangxin Chen and Tatyana Y. Savchuk, Analysis of the multiscale finite element method for nonlinear and random homogenization problems, SIAM J. Numer. Anal. 46 (2007/08), no. 1, 260-279, DOI 10.1137/060654207. MR2377263 (2008k:35020)

[19] Lolimar Díaz and Raúl Naulin, A proof of the Schauder-Tychonoff theorem, Divulg. Mat. 14 (2006), no. 1, 47-57 (English, with English and Spanish summaries). MR2586360 (2010k:47118)

[20] Jim Douglas Jr. and Todd Dupont, A Galerkin method for a nonlinear Dirichlet problem, Math. Comp. 29 (1975), 689-696. MR0431747 (55 \#4742)

[21] Weinan E and Bjorn Engquist, The heterogeneous multiscale methods, Commun. Math. Sci. 1 (2003), no. 1, 87-132. MR1979846 (2004b:35019)

[22] Weinan E, Pingbing Ming, and Pingwen Zhang, Analysis of the heterogeneous multiscale method for elliptic homogenization problems, J. Amer. Math. Soc. 18 (2005), no. 1, 121-156, DOI 10.1090/S08940347-04-00469-2. MR2114818 (2005k:65246)

[23] Yalchin Efendiev and Thomas Y. Hou, Multiscale finite element methods, Surveys and Tutorials in the Applied Mathematical Sciences, vol. 4, Springer, New York, 2009. Theory and applications. MR2477579 (2010h:65224)

[24] Y. Efendiev, T. Hou, and V. Ginting, Multiscale finite element methods for nonlinear problems and their applications, Commun. Math. Sci. 2 (2004), no. 4, 553-589. MR2119929 (2005m:65265)

[25] Yalchin R. Efendiev, Thomas Y. Hou, and Xiao-Hui Wu, Convergence of a nonconforming multiscale finite element method, SIAM J. Numer. Anal. 37 (2000), no. 3, 888-910, DOI 10.1137/S0036142997330329. MR1740386 (2002a:65176)

[26] Yalchin Efendiev and Alexander Pankov, Numerical homogenization of monotone elliptic operators, Multiscale Model. Simul. 2 (2003), no. 1, 62-79, DOI 10.1137/S1540345903421611. MR2044957 (2005a:65153)

[27] Lawrence C. Evans, Partial differential equations, 2nd ed., Graduate Studies in Mathematics, vol. 19, American Mathematical Society, Providence, RI, 2010. MR2597943 (2011c:35002)

[28] Charbel Farhat, Isaac Harari, and Leopoldo P. Franca, The discontinuous enrichment method, Comput. Methods Appl. Mech. Engrg. 190 (2001), no. 48, 6455-6479, DOI 10.1016/S0045-7825(01)00232-8. MR1870426 (2002j:76083)

[29] Leopoldo P. Franca, Alexandre L. Madureira, Lutz Tobiska, and Frédéric Valentin, Convergence analysis of a multiscale finite element method for singularly perturbed problems, Multiscale Model. Simul. 4 (2005), no. 3, 839-866 (electronic), DOI 10.1137/040608490. MR2203943 (2006k:65316)

[30] Leopoldo P. Franca, Alexandre L. Madureira, and Frederic Valentin, Towards multiscale functions: enriching finite element spaces with local but not bubble-like functions, Comput. Methods Appl. Mech. Engrg. 194 (2005), no. 27-29, 3006-3021, DOI 10.1016/j.cma.2004.07.029. MR2142535 (2006a:65159)

[31] L. P. Franca and A. Russo, Deriving upwinding, mass lumping and selective reduced integration by residual-free bubbles, Appl. Math. Lett. 9 (1996), no. 5, 83-88, DOI 10.1016/0893-9659(96)00078-X. MR1415477 (97e:65121)

[32] Christopher Harder, Diego Paredes, and Frédéric Valentin, A family of multiscale hybrid-mixed finite element methods for the Darcy equation with rough coefficients, J. Comput. Phys. 245 (2013), 107-130, DOI 10.1016/j.jcp.2013.03.019. MR3066201 
[33] Patrick Henning, Convergence of MSFEM approximations for elliptic, non-periodic homogenization problems, Netw. Heterog. Media 7 (2012), no. 3, 503-524, DOI 10.3934/nhm.2012.7.503. MR2982460

[34] Thomas Y. Hou and Xiao-Hui Wu, A multiscale finite element method for elliptic problems in composite materials and porous media, J. Comput. Phys. 134 (1997), no. 1, 169-189, DOI 10.1006/jcph.1997.5682. MR1455261 (98e:73132)

[35] Thomas Y. Hou, Xiao-Hui Wu, and Zhiqiang Cai, Convergence of a multiscale finite element method for elliptic problems with rapidly oscillating coefficients, Math. Comp. 68 (1999), no. 227, 913-943, DOI 10.1090/S0025-5718-99-01077-7. MR1642758 (99i:65126)

[36] Alexandre Madureira, Abstract multiscale-hybrid-mixed methods, Calcolo, to appear.

[37] Alexandre L. Madureira, A multiscale finite element method for partial differential equations posed in domains with rough boundaries, Math. Comp. 78 (2009), no. 265, 25-34, DOI 10.1090/S0025-5718-0802159-5. MR2448695 (2010a:65243)

[38] Pingbing Ming and Xingye Yue, Numerical methods for multiscale elliptic problems, J. Comput. Phys. 214 (2006), no. 1, 421-445, DOI 10.1016/j.jcp.2005.09.024. MR2208685 (2006j:65359)

[39] J. Pousin and J. Rappaz, Consistency, stability, a priori and a posteriori errors for PetrovGalerkin methods applied to nonlinear problems, Numer. Math. 69 (1994), no. 2, 213-231, DOI 10.1007/s002110050088. MR1310318 (95k:65111)

[40] Jairo V. de A. Ramalho, Novos métodos de elementos finitos enriquecidos aplicados a modelos de reaçãoadvecção-difusão transientes, D.Sc. Thesis, Laboratório Nacional de Computação Científica, Petrópolis, RJ, 2005.

[41] Giancarlo Sangalli, Capturing small scales in elliptic problems using a residual-free bubbles finite element method, Multiscale Model. Simul. 1 (2003), no. 3, 485-503 (electronic), DOI 10.1137/S1540345902411402. MR2030161 (2004m:65202)

[42] S. Tokarzewski and I. Andrianov, Effective coefficients for real non-linear and fictitious linear temperature-dependent periodic composites, Internat. J. Non-Linear Mech. 36 (2001), no. 1, 187-195, DOI 10.1016/S0020-7462(00)00012-3. MR1783661 (2001i:80011)

[43] H. M. Versieux and M. Sarkis, Numerical boundary corrector for elliptic equations with rapidly oscillating periodic coefficients, Comm. Numer. Methods Engrg. 22 (2006), no. 6, 577-589, DOI 10.1002/cnm.834. MR2235030 (2007d:65117)

[44] Henrique Versieux and Marcus Sarkis, A three-scale finite element method for elliptic equations with rapidly oscillating periodic coefficients, Domain decomposition methods in science and engineering XVI, Lect. Notes Comput. Sci. Eng., vol. 55, Springer, Berlin, 2007, pp. 763-770, DOI 10.1007/978-3-54034469-8_95. MR2334173

[45] Jinchao Xu, Two-grid discretization techniques for linear and nonlinear PDEs, SIAM J. Numer. Anal. 33 (1996), no. 5, 1759-1777, DOI 10.1137/S0036142992232949. MR1411848 (97i:65169)

[46] Tao Yu and Xingye Yue, Residual-free bubble methods for numerical homogenization of elliptic problems, Commun. Math. Sci. 9 (2011), no. 4, 1163-1176, DOI 10.4310/CMS.2011.v9.n4.a12. MR2901822 